\title{
Vacuum Switching Experiments at California Institute of Technology
}

\author{
ROYAL W. SORENSEN* \\ Fellow, A. I. E. E.
}

\begin{abstract}
Synopsis.- Successful experiments in swilching or breaking a circuit in a high vacuum have been made at the California Institute of Technology. This paper is a report on three sets of these experiments which extended over a period of three years. The conclusions drawn from the experiments may be summed up in the statement that vacuum breakers of laboratory type have been successful in breaking circuits and offer a possible solution of the circuit-breaker problem.
\end{abstract}

E

XPERIMENTS on breaking an electrical circuit in a high vacuum have been made during the last three years at the California Institute of Technology in connection with the study of switching highvoltage, high-power circuits. These experiments were undertaken as a result of the well-known limitations of oil circuit breakers. A large number of tests was made on high-vacuum breakers of laboratory type. Some very promising results were obtained in interrupting large currents.

When these experiments were suggested, the question immediately presenting itself was: Will the vacuum maintain itself at the time the arc is formed between the separating metallic parts of an opening switch?

This doubt was quite generally substantiated by the commonly recognized theory of the electric arc, ${ }^{1} \mathrm{viz}$, that the maintenance of an arc is dependent upon the giving out of thermions from hot spots on the electrodes between which the arc is formed, with the attendant vaporization of the metal. If this were true, a large current could not be interrupted in a vacuum because the formation of even a small amount of gas would reduce the vacuum and cause it to become a conducting vacuum rather than an insulating vacuum.

The fact of the matter, however, is that if the vacuum is sufficiently high and all adsorbed gases have been removed from the metal electrodes, very large currents can be broken without formation of enough vapor to maintain an are.

Dr. R. A. Millikan has shown ${ }^{2}$ that, with cold electrodes suitably prepared, millions of volts of potential gradient are required to obtain discharges of any kind between metal surfaces. He has also worked out with much care the conditions necessary for denuding metal surfaces of gases and preventing the impairment of the vacuum through the evolution of gases. A. Janitzky ${ }^{3}$ also has reported experiments showing that currents will not flow across the space between cold electrodes in a vacuum provided the electrodes have been completely outgassed.

*Both of the California Institute of Technology, Pasadena, Calif.

1. For references, see Bibliography.

Presented at the Pacific Coast Convention of the A. I. E. E., Salt Lake City, Utah, Sept. 6-9, 1926.
HALLAN E. MENDENHALL*

Associate, A. I. E. E.

The results show that switching in vacuum affords the advantages of no pitting of contacts, quick break, the arc always going out on the first half-cycle, small voltage rise across the switch, and small distance of travel necessary for the switch blades.

Making the vacuum switch practical calls for a solution of the problem of making commercial apparatus with vacuum-tight joints, and the elimination of the use of liquid air with the vacuum pump.

According to the older theory, it would seem that considerable vapor might be formed in the vacuum on breaking a circuit. P. Charpentier ${ }^{4}$ has given the following equation:

$$
W=0.07 E I t
$$

as the equation for the energy to be dissipated in an oil switch at the time of opening. In this equation, $E=$ voltage, $I=$ current, and $t=$ the time in seconds between the initial separation of the switch contacts and the complete extinguishing of the arc. Charpentier's experiments, and also those made by Swiss engineers in 1915 and 1916, indicate that this energy is used in vaporizing oil at the rate of $46.5 \mathrm{cu}$. $\mathrm{cm}$. per $\mathrm{kw}$-sec. Some of the tests made on oil switches show the vaporization of smaller amounts of oil per $\mathrm{kw}$-sec. and also show power factors of less than 0.07 across the switch at the time of interruption. Applying Charpentier's equation to a single-pole switch opening a 15,000-volt, 100-ampere, 50-cycle circuit, we find that the switch must dissipate $1.05 \mathrm{kw}$-sec. if it opens on the first halfcycle. Assuming as an extreme case, for the vacuum switch, all of this energy available to vaporize copper at the switch blades, we find that it would vaporize approximately one-fifth gram. This amount of copper turned into vapor would reduce an insulating vacuum in a container of considerable size to a vacuum which would be conducting for 15,000 volts applied between electrodes extending into the container.

However, the later theories to which reference has been made indicate that such an amount of vapor will not be formed provided the vacuum is high and the electrodes are free from gas.

Therefore, in making the experiments, the prime requisites were to have the electrodes entirely free of adsorbed gases and to obtain a good vacuum. Dr. Millikan was immediately interested in the proposal of the tests and placed at our disposition the facilities and high-vacuum experience of the Norman Bridge Laboratory; also he cooperated in the development of the switch by making many valuable suggestions and by assigning to the work two graduate students of the physics department, H. E. Mendenhall and Russell Otis.

Three switches were developed and tested. The first 
switch is shown in Fig. 1. It consists of a glass envelope with two fixed electrodes as shown, separated by one-half inch. These have crescent-shaped contactsurfaces, $a$ and $b$, as shown. The contact area of each of the fixed terminals is $1 / 8 \mathrm{sq}$. in. The circuit is closed by a flat circular copper disk resting upon them with no contact pressure other than the weight of the disk, to which is attached a light plunger. The switch is opened in operation by a
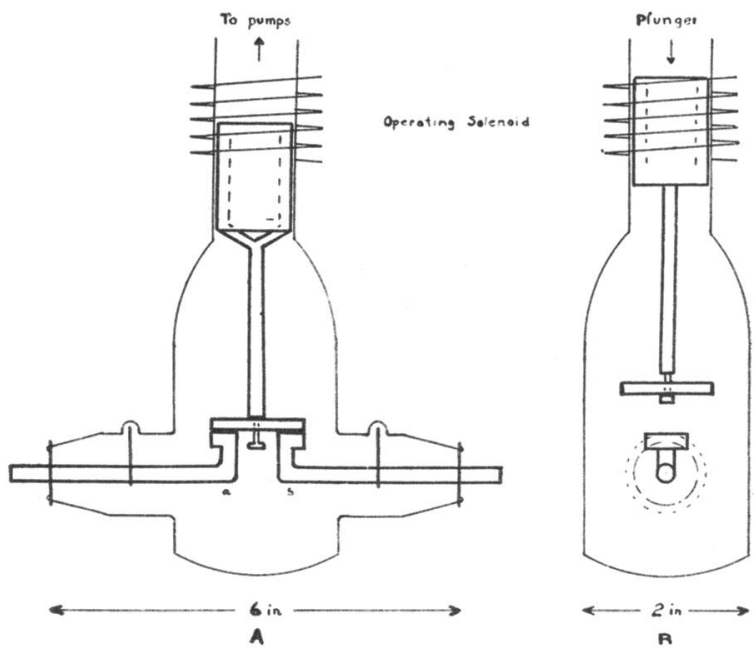

Fig. 1-Cross-Section Views of Switch No. 1

solenoid which, when energized, raises the plunger. In interrupting the circuit, the bridging circuit contact is raised $1 / 2 \mathrm{in}$. by the solenoid. This type of construction gives two breaks in series when the switch is open.

Vacuum-tight joints between the lead-in conductors

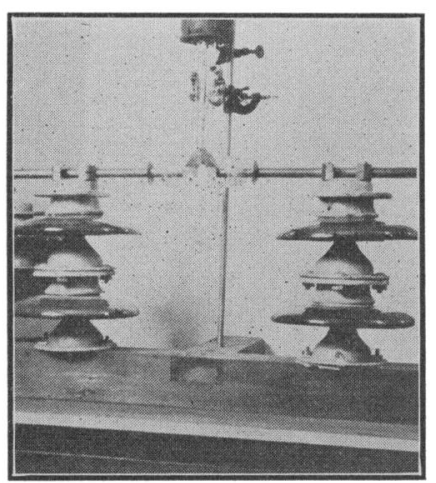

Fig. 2-Vactum Switch No. 2

and the glass envelope of the switch were easily obtained by means of W. G. Houskeeper's disk seals. ${ }^{5}$ This switch was evacuated down to $10^{-6}$ centimeters of mercury pressure. An initial test was made by using this switch to interrupt currents up to 125 amperes at 110 volts d-c. The results were encouraging, and the switch was connected to an a-c. supply and the test repeated with very satisfactory results, the interruption of current being accomplished with less arcing than occurred when direct current was used.

The switch was then successively used on a-c. circuits for 220 volts, 2300 volts, and 15,000 volts. The load in every case was a single-phase load connected and disconnected by means of the switch used as a singlepole switch. There was no apparent difference in the operation of the switch at the different voltages with the exception that the switch was not properly designed to guard against arcing over the outside at 15,000 volts. This trouble was eliminated by immersing the switch in oil. When so immersed the switch was operated many times as a single-pole switch to interrupt 100 amperes at 15,000 volts. Every operation was successful.

The terminals of this switch, however, were very

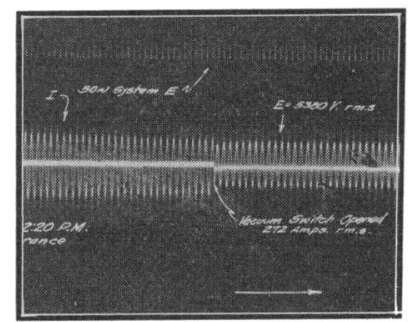

Fig. 3-Vacudm Switch Interiupting 272 Amperes at 5380 Volts

small and therefore a second switch having terminals with more contact surface and leads of greater carrying capacity was built. Fig. 2 shows switch No. 2. This switch was constructed in the same manner as switch No. 1 , but is larger and has better contacts, the bridge being made of spring-copper laminations. When the switch is closed, the edges of the laminations are held

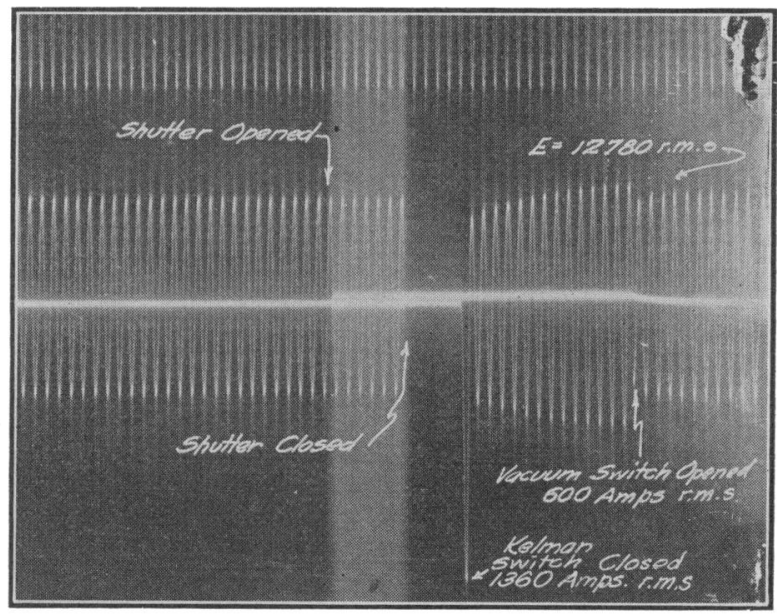

Fig. 4-Vacuum Switch Interrupting 600 Amperes at 12,780 VOLTS

against the fixed contacts by the weight of the bridge and its lifting solenoid, the total weight being two pounds. The contact area of each fixed terminal is $3 / 4 \mathrm{sq}$. in., the distance between the fixed terminals being one inch. In interrupting circuit the bridge is raised one inch.

This switch was given laboratory tests on a 15,000volt, single-phase circuit providing currents up to 120 
amperes at this voltage. The switch was operated as a single-pole switch to open and close this circuit more than 500 times without showing any burning of the switch contacts. It was then sealed off from the vacuum pump and allowed to stand in the laboratory for three months, during which time it was tested occasionally to determine its condition. At the end of the three months the switch was taken to the Torrence substation of the Southern California Edison Company and used to open short circuits made on a synchronous

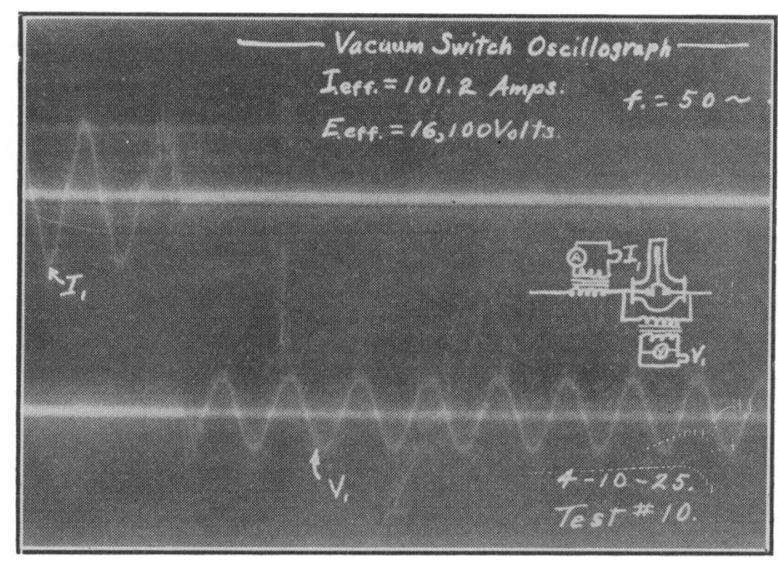

Fig. 5-Oscillogram Showing Current Opened by Vacuum Switch and Voltage Across Switch at Opening

$I_{\text {eff }}=101.2$ amperes $\quad E_{\text {eff }}=16,100$ volts $f=50$ cycles

condenser just as the condenser was disconnected from the Edison distribution system. The currentwas supplied to the switch from the condenser through step-up transformers. The switch repeatedly opened the singlephase short circuit thus provided without any failure

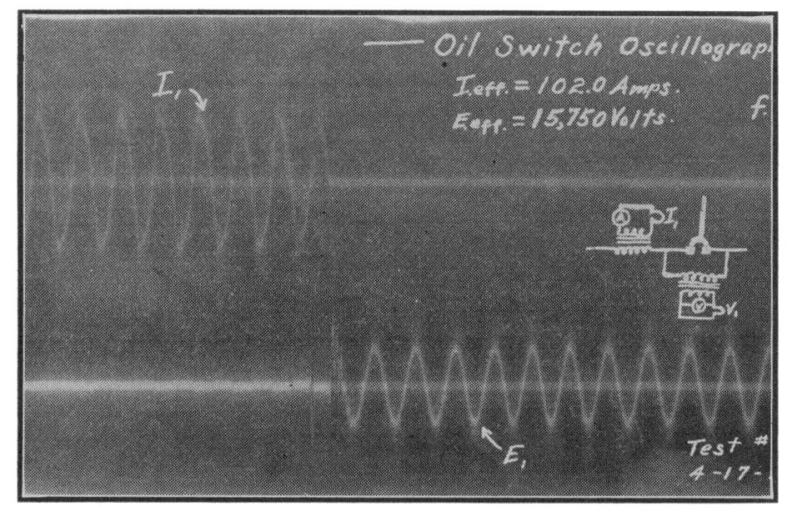

Fig. 6-Oscillogram showing Current Opened by Oil Switch and Voltage Across Switch at Opening

Note that 12 cycles are between initial contact separation and extinguishing of arc

$I_{\text {eff }}=102$ amperes $E_{\text {eff }}=15,750$ volts $f=50$ cycles

to interrupt the circuit or any burning of the switch contacts. Figs. 3 and 4 show oscillograms of switch No. 2 opening 272 amperes at 5380 volts and 600 amperes at 12,780 volts, respectively.

Figs. 5 and 6 show oscillographic records of vacuum switch No. 2 and a standard make of oil switch opening the same circuit on a load of 100 amperes at 15,000 volts. The tests were made under conditions as nearly identical as possible, and within a few minutes of each other. It will be noted from these graphs that the rise in voltage when the circuit is opened with the oil switch is greater than when the circuit is opened with the vacuum switch. The oil switch in a large number of tests failed to open the circuit on the firsthalf-cycle, while the vacuum switch always opened the circuit on the first half-cycle. An examination of a number of oscillograph records for oil switches and for the vacuum switching showed that when the circuit was opened the rise in voltage above normal circuit voltage was higher for the oil switch than for the vacuum switch. The klydonograph ${ }^{6}$ was used in some of the switching tests to record any high-frequency surges that might occur.

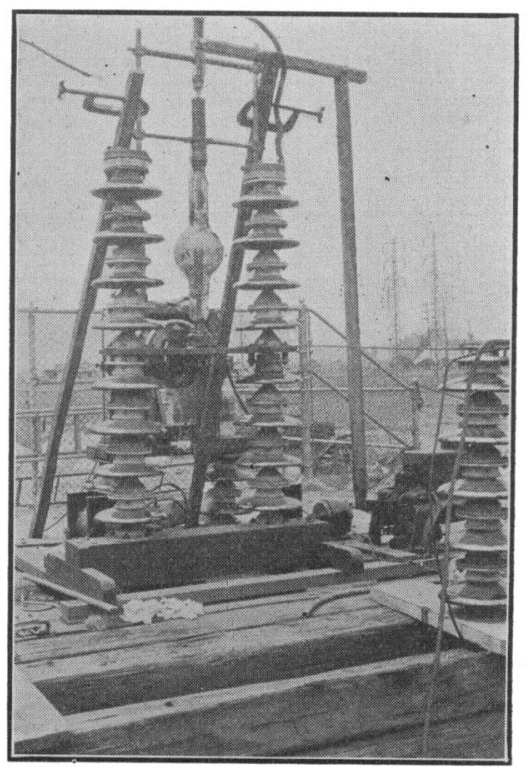

Fig. $\quad 7-V_{\text {acuum }}$ Switch No. 3

In no case did the instrument indicate voltages much above normal.

Following these tests, switch No. 3 shown in Fig. 7 was constructed. The figure shows the switch in the closed position. Switch No. 3 was constructed primarily to overcome the disadvantage, in switches Nos. 1 and 2, of having all moving parts sealed inside the glass envelope of the switch, a condition requiring the operating solenoid to be kept energized when the switch is open, unless a rather intricate locking mechanism also be installed inside the switch to hold it open.

In switch No. 3, the moving contact is of the bayonet type, the bayonet sliding into a cylindrical socket. The bayonet is a $3 / 4$-in. copper rod projecting into the socket when closed so as to give a contact length of one in., the total contact surface obtained in this way being $2.3 \mathrm{sq}$. in. With this construction, there is only a single break, the contacts opening so as to separate them a distance of one in. when the switch is completely open. 
The single break appeared to function as well as the double break used in switches 1 and 2. The switch was operated by a standard switch-operating mechanism borrowed from an oil switch. With such an arrangement, the switch can be left open or closed at will. Vacuum-tight joints for the lead-in conductors of this switch were made by cementing to the glass envelope metal caps attached to the leads and forming a part of the leads.

After some preliminary testing in the laboratory, this switch was taken to the Laguna-Bell substation of the Southern California Edison Company and used as a single-pole switch to open single-phase short circuits on a 30,000-kv-a., synchronous condenser. In performing the tests, the synchronous condenser was brought up to speed on the distribution system of the Edison Company, disconnected from the system and immediately short-circuited through the switch. The condenser used was a 6600-volt, three-phase, Westinghouse condenser which, for the purpose of testing the switch, was connected to the switch through step-up transformers by means of which voltages across the switch as high as 41,500 volts were reached. Fig. 8

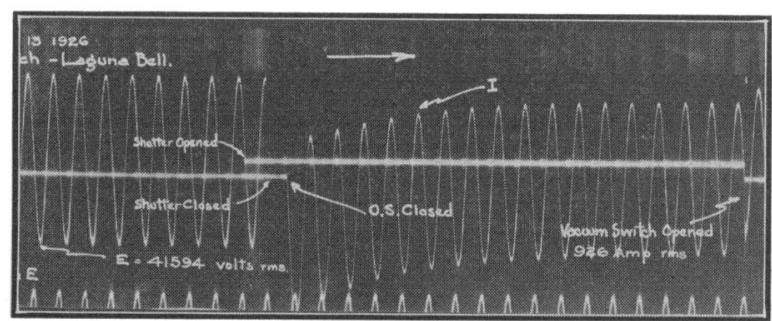

Fig. 8-Vacuem Switch Opening 926 Amperes at 41,594 VOLTS

shows an oscillogram for this switch interrupting 926 amperes at 41,500 volts.

A noticeable feature of the vacuum-switch tests is that every oscillographic record shows that the are produced at the opening of the switch is extinguished at the end of the first half-cycle after the separation of the contacts. Only the very best oil-switch operations give this result.

The absence of any pitting of switch contacts and the fact that the vacuum is not reduced appreciably when the switch is in operation is evidence that very little of the energy dissipated when the switch is opened is used in vaporizing metal from the contacts.

When the contacts separate, there is a visible arc, just as when a switch is opened in air or oil. The magnitude of this arc, however, is much less than that of an arc made by like values of voltage and current in air or oil. This is to be expected because there is nothing in a vacuum switch to burn or to support combustion, as is the case when a switch is opened in oil or air.

The action of the arc in vacuum also indicates a doubt as to the soundness of the theory that an are to be maintained must be supported by thermions emanating from hot spots on the electrodes between which the arc is formed. J. Slepian ${ }^{7}$ has shown that an are is probably formed near the surface of metal electrodes by very high temperatures caused by the concentration of electric current in the gas immediately surrounding the electrodes. The experiments at California Institute of Technology show that the vacuum switch, when opened, fails to interrupt an electric circuit if the metal forming the contacts has not been freed of theadsorbed gases; that is, gases adhering to the surface of the metal.

The results of these experiments cannot be taken as conclusive evidence that a new type of electric switch has been developed, because the limits of performance have not been determined and there are many problems relating to details that must be solved to make the switch practical. The switch, however, was never the limiting factor in any of the tests made. There is, therefore, certainly sufficient encouragement to warrant further investigation of the subject for the purpose of determining the fundamentals of switching phenomena, if for no other reason. Also, we have the encouraging fact that many practical devices in use today presented, in the early stages of their development, obstacles which appeared greater than those which these tests indicate.

The authors of this paper are indebted to those already mentioned as having a part in the program, and in addition, to graduate students in the Department of Electrical Engineering, J.J. DeVoe, J.H. Hamilton, and F. C. Lindvall, for assisting in laboratory and field tests; to Julius Pearson and William Clancy for their skill in doing the machine work and glass blowing; and also to the many members of the Southern California Edison Company who made provision for having switches Nos. 2 and 3 tested on the Edison system and helped in making the tests.

\section{Bibliography}

1. K. T. Compton, "Theory of the Electric Arc," Phys. Rev., 21, p. 266, (1923).

2. Millikan and Shackelford, Phys. Rev., 15, p. 239, (1920); also Millikan and Eyring, Phys. Rev., 27, p. 51, (1926).

3. Alexander Janitzky, "Uber die Bedentung des Gasgehalts von Metallen fur einige elektrische Erscheinungen," Zeitschrift fur Physik, 31, S277, (1925).

4. P. Charpentier, "Dimensions, Construction and Design of Oil Switches," Revue General de Lectrique, May 5, 1923, p. 737.

5. W. G. Houskeeper, "Sealing Base Metals through Glass," A. I. E. E. Journal, 42, pp. 954-960, (Sept. 1923).

6. J. H. Cox and J. W. Legg, "The Klydonograph and Its Application to Surge Investigation," A. I. E. E. JoURNAL, 44, (Oct. 1925).

7. J. Slepian, "The Transition from Glow to Are Discharge at Atmospheric Pressure," Journal of the Franklin Institute, 201, p. 79, (Jan. 1926). 


\section{Discussion}

Joseph Slepian: I was surprised when I read in Professor Sorensen's paper that he found very little pitting or vaporizing of the electrodes. It seems to me that this, if true, must call for some radical revision in our ideas of electric ares.

As Professor Sorensen mentions in his paper, the most widely held theory of the ares requires that the cathode be maintained at a temperature sufficiently high for thermionic emission. I agree with Professor Sorensen that this theory is probably not correct and that a hot cathode is not essential for an are. It is possible to have a high-current are with a cold cathode.

Some most convincing evidence on this point published in the $Z$ eitschrift fur Physik is due to H. Solt, who caused a 10-ampere are to move over a copper surface so rapidly that the copper did not get hot at any pcint.

I have repeated this experiment and I have caused ares with as high as 20,000 amperes to move so rapidly over a copper ring that there was no visible melting of any kind.

But while it seems that a hot cathode is not necessary it does appear that in order to carry heavy current at low voltage a considerable pressure of gas or vapor of some sort is necessary. This gas or vapor would have to be supplied from the electrodes, and presumably in a successful operation of the switch this metal vapor would condense so rapidly at the zero of current that the high vacuum would be restored suffieiently to withstand the line voltage. But now, however, going by theory and by experience, to carry an are of several thousand amperes will require a vapor pressure in the order of millimeters, and if the electrodes are to supply this vapor for a full half-cycle, serious consumption of the electrodes will result.

If only a small amount of vapor in fact is formed, it would seem that the discharge must be of some very high-voltage form. I should like therefore to ask Professor Sorensen what the voltage on the arc was in his vacuum-switch experiments.

I should appreciate also some furcher information concerning the last switch mentioned in his paper, particularly how the moving electrode is brought into the vacuum tube.

D. C. Prince: The oscillograms shown in this paper indicate that in some way current is carried between the electrodes after they have separated until the termination of the half-cycle. It is not necessary to assume that this current is thermionic.

The mechanism used to explain the cathode spot in the mercury rectifier might be applicable to this phenomena. It is briefly as follows: Electrons may be drawn from a relatively cool metal by an electric field of some millions of volts per centimeter. These electrons proceed until they strike neutral gas molecules, which they ionize. The positive ions so formed progress toward the cathode and set up, in this case copper, a high space-charge gradient required to extract further electrons from the cathode material. The positive ions striking the copper surface heat it to the vaporizing point so that copper vapor is given off and is present to be ionized by the electrons.

In order that this action may take place in the extremely short distance required to give the potential gradient of millions of volts per centimeter, the copper vapor would have to have a pressure of the order of atmospheres in the neighborhood of the cathode. Upon the termination of a half-cycle, the cathode spot would go out and there would be no mechanism to establish a cathode spot on the other electrode. Thus conductivity would be interrupted.

P. H. Thomas: Some years ago in the laboratories of the Cooper Hewitt Electric Company, I made rather extensive researches on the behavior of vacuum apparatus passing alternating currents, and arrived at a practical conception of these phenomena which may be of interest in connection with the further work of the authors. The question as to why the arc behaves as it does in a high vacuum is fundamental, of course, in considering what it is likely to do with heavier currents.

My conception of this phenomenon in 1906 is covered in a paper $^{1}$ presented before the A. I. E. E., May 31st, 1906, and is briefly as follows:

The electric current in a perfect vacuum is taken to be a stream of electrons, as with the bombardment in an X-ray tube. Once in the perfect vacuum, these electrons pass without resistance and without giving light or generating heat. To release such electrons carrying electric charges from a cold conducting surface in a perfect vacuum would theoretically require an infinite voltage. If, however, it be assumed that the flow of electrons has once been established (as by the well-known method of separating metallic contacts in the vacuum) it is reasonable to suppose that at the point on the cathode surface where the stream issues, an open door, so to speak, may be established in such a way that the assumed perfect surface of the metal is locally changed to give physically a gradual transition from a solid state to a gaseous state, then a comparatively small voltage would be sufficient to move electrons from the cathode to the vacuum space in accordance with the observed phenomena. To put it another way, the physical surface tension of the metal, taken in an electrical sense, is punctured at a certain spot by the passage of a certain amount of current and cannot heal until the stream stops, as at the zero point of an a-c. wave. As a matter of fact, if the current from a solid cathode once established in a perfect vacuum be gradually decreased in strength, a point will be reached at a strength of a few amperes at which it will snap out violently. In fact, by using a series inductance in a d-c. circuit, a most excellent Wehnelt interrupter may be obtained, giving exceedingly abrupt high-voltage impulses.

According to this view the cathode spot from which the current issues in a perfect vacuum,--even from a solid electrode,--need not be a hot spot and, as a matter of fact, the phenomenon is much more easily explained if it be assumed cold. Thece is of course a brilliant light spot showing the excitation of the atoms at that point but the actual rise of temperature is probably small. This action is much clearer with mercury cathodes which cannot become heated on account of their power to evaporate but which exhibit the same switch action as the solid cathode.

This does not mean that arcs may not be fed by hot spots on eathodes, in the presence of gases. It seems probable that the critical feature of the vacuum switch which easily drops an arc at the zero point of the wave is the cold cathode, for such a cathode has no tendency to hold over the zero point, while the reason the are in air does not drop out easily at the zero point is that the cathode spot is hot enough to emit electrons and stays so over the zero point.

The reason why the vaporization of copper at the cathode does not impare the vacuum would appear to be that copper is naturally a solid at ordinary temperatures and that any vaporized copper would immediately solidify and become inert. I believe that this type of circuit breaker will be very effective up to the point where in operation the vacuum does not deteriorate or the electrodes actually develop hot spots.

As a matter of fact, at the time of writing of the above paper, I operated vacuum switches in the laboratory for the purposes of investigation both with mercury and with solid electrodes, but not with such large quantities of energy nor so comprehensively as the authors.

Some of the specifications of patents on vacuum apparatus filed about this time and assigned to the Cooper Hewitt Electric Company would be of interest in this connection.

R. W. Sorensen: Dr. Slepian asked two or three definite questions. The first two switches were very poorly designed for practical operation; that is, the contacts were poor as judged by switch engineers and the solenoid coils around the neck of the switch, used to raise the plunger, were crude ones built by students inexperienced in the design of solenoids. These solenoids were weak and operated the plunger with a hesitating

1. Some Fundamental Characteristics of Mercury Vapor Apparatus, A. I. E. E. Trans., 1906, p. 601. 
action. This uncertain action I am sure was the cause of many of the irregularities in the oscillograms made for switches 1 and 2. The poor operating mechanism in these switches was particularly noticeable in closing them. Switches No. 1 and No. 2 were closed by gravity when the solenoid cireuit was opened. Such action of course caused the plunger to rebound several times at closing, thus causing an areover each time the switch was closed, a very poor arrangement for a switching device.

We have shown no oscillograms for the switch closing, because there is no question as to its operation on closing. When the switch is closing, the contacts are absolutely cold and the insulation resistance of the vacuum space between switch terminals is so high that no arc can form between terminals when the switch is actually closed. This is frequently the case in an oil switch. Examination of the small switch shows that with all the ill treatment of the contacts for reasons just mentioned, there has been no appreciable pitting of the contacts. Switch No. 1 and also No. 2 has each operated according to our records more than 500 times. There were many additional operations of which no record was made. As stated in the paper, switch No. 3 is unique because in it we have found a means whereby we can bring the operating rods through the switch envelope and at the same time maintain a vacuum-tight envelope. The paper calls attention to the fact that switch No. 2 stood in the laboratory two months, disconnected and sealed off from the vacuum pump, before it was tried out at the Torrence Substation of the Southern California Edison Company.

Also I have used in calculations higher values for the amount of copper to be vaporized by a given amount of energy. The latest international tables of physical constants give $467 \mathrm{kw}$-sec. of energy required per gram molecule of copper, a much higher figure than the one we had been in the habit of using.

H. E. Mendenhall: Questions have been asked as to the theory of the vacuum switch but our work is in such an early experimental stage that we are not positive as to the exact mechanism of the high-vacuum are.

Mr. Prince has suggested that the electrons to maintain the vacuum are may be pulled out of the metal by very high electric fields as in the mercury rectifier. Dr. Slepian has asked what the voltage drop across the are is. The voltage between the contacts at separation rises momentarily to some value below the maximum value of the voltage wave and then during the time of the are there is apparently very little drop across the are (less than in an oil switch). The are goes out at the end of the first halfcycle, at which time the voltage rises to a value less than 50 per cent above normal, the corresponding value for the oil switch being somewhat higher. The work of Millikan and Eyring has shown that potential gradients as high as 4,000,000 volts per $\mathrm{cm}$. are required to pull electrons out of metals up to $700 \mathrm{deg}$. cent. It is possible that the first electrons for starting the are are pulled from the metal in the manner described by them, consuming a considerable portion of the voltage drop representing the energy dissipation in the are, the balance of the drop going to vaporize and ionize enough copper atoms to establish conditions for thermionic emission for one half-cycle. These conditions disappear with the recombination of the extremely short-lived metal ions and the reverse of the direction of current flow between switch terminals which from the physics point of view are electrodes whose separation distance is increasing during the opening of the switch.

We were very much interested in some work published by Dr. Slepian in which he advocated a rather new theory for the transition from glow to are discharges. Instead of this transition being due to thermions from within the electrode, he reasoned that temperatures sufficiently high existed in the gas surrounding the electrodes to produce thermal ionization of the gas. From our work, in which we remove as much gas as possible and are thus able to extinguish the arc, we have concluded that the gases within the metal and absorbed on its surface in a layer perhaps only one molecule thick play an essential part in the maintenance of an are.

We believe that the difference between our results and those mentioned by Dr. Slepian are due to a difference in the degree of vacuum attained in the two cases. We have used the latest high-vacuum technique so that although we have a so-called high vacuum of $10^{-6} \mathrm{~mm}$. of mercury pressure, we have actually removed relatively few gas molecules, but we have increased very materially the mean free path of the molecules or the average distance one molecule will travel before it collides with another molecule. The importance of this factor becomes evident when we consider that an ion or electron may travel across the short distance between the electrodes of the switch in high vacuum without colliding with another molecule and thus ionization of additional gas molecules by collision is not likely to occur.

Mr. Thomas in his discussion assumes it is physically possible to obtain a perfect vacuum but we have already pointed out that there are numerically more gas molecules left in the evacuated chamber than have been removed.

The cathode spot which furnished electrons for one half-cycle becomes the anode when the voltage reverses and the new cathode is a relatively cold cathode so that electron emission from its surface cannot be started without intense bombardment by positive metal ions. We think that these same ions have had time to become ineffective due to recombination since their mean life is of the order of $10^{-8}$ sec.

We are not yet decided as to how all of the stored energy of the electric and magnetic fields, as roughly evaluated by Charpentier's formula, is dissipated, but our future program includes experiments which should determine better the principles involved in the operation of electric switches. 